\title{
Frejol castilla (Vigna unguiculata L. Walp) como ingrediente en raciones de crecimiento-engorde de cuyes (Cavia porcellus) mejorados
}

\author{
COWPEA (VIGNA UNGUICULATA L. WALP) AS INGREDIENT IN GROWING-FATTENING RATIONS OF \\ IMPROVED GUINEA PIGS (CAVIA PORCELLUS)
}

Katherine Choez A. ${ }^{1,2}$, Víctor Ravillet S. ${ }^{1}$

\section{Resumen}

\begin{abstract}
El objetivo del estudio fue determinar la respuesta productiva y económica de cuyes mejorados (Linea Perú tipo I) alimentados con raciones conteniendo diferentes niveles de frejol Castilla (Vigna unguiculata L. Walp). El estudio se llevó a cabo en una granja familiar de Lambayeque, Perú, con 40 cuyes machos destetados de 15 días y $387.5 \mathrm{~g}$ de peso inicial, bajo un diseño completamente randomizado. Los cuyes se distribuyeron en cuatro tratamientos: T0, T1, T2 y T3 con 0, 10, 20 y 30\% de frejol Castilla en las raciones, respectivamente. El concentrado fue ofrecido ad libitum, además de $150 \mathrm{~g}$ de alfalfa por cuy/día. Luego de siete semanas, la ganancia de peso varió entre 10.3 y $12.0 \mathrm{~g} / \mathrm{d}$ áa, el consumo en concentrado varió entre 38.8 y $47.2 \mathrm{~g} / \mathrm{cuy} /$ día y el rendimiento de carcasa varió entre 65.0 y $66.6 \%$, sin diferencias significativas entre tratamientos. La mejor conversión alimenticia del concentrado se observó en T2 (3.6). El mejor mérito económico fue, asimismo, en T2, el cual presentó $20.7 \%$ de mejora económica adicional que T0. En conclusión, el frejol Castilla en el nivel de $20 \%$ en las raciones de crecimiento-engorde de cuyes mejorados mejora el índice de conversión alimenticia y el mérito económico.
\end{abstract}

Palabras clave: cuy; alimentación; frejol Castilla

\section{Abstract}

The objective of the study was to determine the productive and economic response of improved guinea pigs (Peru type I) fed with rations containing different levels of cowpea (Vigna unguiculata L. Walp). The study was conducted in a family farm of Lambayeque, Peru, using 40 weaned male guinea pigs of 15 days and $387.5 \mathrm{~g}$ of initial weight, under a completely randomized design. The guinea pigs were distributed in four

\footnotetext{
${ }^{1}$ Laboratorio de Nutrición Animal, Facultad de Medicina Veterinaria, Universidad Nacional Pedro Ruiz Gallo, Chiclayo, Perú

${ }^{3}$ E-mail: kathyvet3@hotmail.com
}

Recibido: 3 de mayo de 2017

Aceptado para publicación: 20 de septiembre de 2017 
treatments: T0, T1, T2 and T3 with 0, 10, 20 and 30\% of cowpea in the rations, respectively. The concentrate was offered ad libitum, plus $150 \mathrm{~g}$ of alfalfa per animal/day. After seven weeks, bodyweight gain ranged from 10.3 to $12.0 \mathrm{~g}$ /day, concentrate consumption varied between 38.8 and $47.2 \mathrm{~g} / \mathrm{animal} /$ day and carcass yield ranged between 65.0 and $66.6 \%$, without significant differences between treatments. The best feed conversion of the concentrate was observed at T2 (3.6). The best economic merit was also in T2, which presented $20.7 \%$ extra income than T0. In conclusion, cowpea at the level of $20 \%$ in the growing-fattening rations of improved guinea pigs improves the rate of feed conversion and economic merit.

Key words: guinea pig; feeding; cowpea

\section{INTRODUCCIÓN}

La crianza de cuyes (Cavia porcellus) es una actividad que paulatinamente ha ocupado un importante espacio dentro de la actividad pecuaria, partiendo de la premisa que es una especie que tiene origen andino y cuyo consumo se ha incrementado en la población urbana (Cruz y Ortiz, 2010). Además, la aceptación y demanda de la carne de cuy viene adquiriendo mayor interés, tanto a nivel nacional como internacional, por sus cualidades nutricionales, como son el bajo nivel de ácido úrico, alto contenido proteico y bajo contenido de grasa (colesterol) (Guerra, 2009; Fajardo, 2011), en comparación con otras carnes.

Se han desarrollado diversos trabajos de investigación en la alimentación del cuy (Chauca, 1997) buscando obtener alimentos adecuados a sus requerimientos nutritivos para lograr óptimos niveles de producción (Rico y Rivas, 2003). El alimento concentrado complementa una buena alimentación, pero la utilización de ingredientes tradicionales encarece los costos de la ración debido a que, generalmente, tienen un alto costo en el mercado (Moncayo, 2012; Ramírez, 2015), debiéndose buscar insumos alternativos accesibles desde el punto de vista económico y nutricional.
La agricultura nacional ha sido reorientada a la exportación y a la agroindustria, generándose grandes volúmenes de subproductos y residuos que podrían ser utilizados en la alimentación animal (Figueroa y Sánchez, 1997). En el caso particular de Lambayeque. Perú, se viene cultivando el frejol (frijol) Castilla o caupí (Vigna unguiculata L. Walp) a gran escala, donde luego de su procesamiento quedan granos pequeños y quebradizos no aceptados para su comercialización y que merecen ser evaluados como parte integrante de la ración alimenticia en animales domésticos, incluyendo el cuy.

En base a las consideraciones expuestas, se desarrolló el presente trabajo con el fin de conocer la respuesta productiva y económica de cuyes mejorados (Linea Perú tipo I) alimentados con raciones concentradas conteniendo diferentes niveles de frejol Castilla.

\section{Materiales y Métodos}

El presente trabajo se realizó en la granja «Santa Elvira», manejada bajo un sistema de crianza familiar, y ubicada en la ciudad de Chiclayo, Perú. La fase experimental tuvo una duración de siete semanas. La muestra de estudio estuvo constituida por 40 cuyes 
machos mejorados línea Perú Tipo I, destetados, de 15 días de edad. Los animales tuvieron un peso inicial de $387.5 \mathrm{~g}$, y fueron distribuidos al azar en cuatro tratamientos experimentales (T0, T1, T2 y T3) de 10 animales cada uno.

Los cuyes fueron alojados en cuatro jaulas de malla metálica, de una dimensión de $1.30 \mathrm{~m}$ de largo, $90 \mathrm{~cm}$ de ancho y $1 \mathrm{~m}$ de altura, cada una con capacidad para 10 cuyes. Estas jaulas se encontraban en un ambiente cerrado, protegido del frío y de las corrientes de aire; además, el ambiente disponía de una buena iluminación natural.

Se elaboraron cuatro raciones balanceadas de acuerdo con los requerimientos nutricionales del cuy (NRC, 1995), utilizando insumos disponibles en la zona, además de la adición del frejol Castilla. La composición de dichas raciones y su valor nutritivo se presentan en el Cuadro 1.

El frejol Castilla empleado fue de descarte, constituido por granos pequeños y quebrados (no aceptados para el comercio en la alimentación humana), adquirido de la Agroindustria San Carlos EIRL (Chiclayo). El insumo fue sometido a molienda y pasado por un tamiz de $1 \mathrm{~mm}$ para su incorporación en tres raciones (T1, T2 y T3) con niveles de 10,20 y $30 \%$, respectivamente.

El concentrado fue proporcionado dos veces al día en cantidades en exceso, además de forraje (alfalfa fresca) en cantidad de $150 \mathrm{~g} / \mathrm{animal} /$ día y agua ad libitum. Se controló el consumo diario de concentrado por diferencia entre la cantidad suministrada y el residuo. El peso vivo fue registrado en forma semanal, en las primeras horas de la mañana, con los animales en ayunas. El control de peso vivo, suministro y rechazo del alimento se hizo con una balanza digital con capacidad de $2.5 \mathrm{~kg}$. y precisión de $1 \mathrm{~g}$. El estudio tuvo una duración de 49 días en que se tomó el peso vivo final. Al término del experimento todos los animales fueron sacrificados, registrándose el peso de la carcasa fresca.
La conversión alimenticia (CA) fue calculada mediante el alimento consumido ( $\mathrm{kg} /$ animal/ periodo) dividido por la ganancia total de peso vivo $(\mathrm{kg})$; el mérito económico (ME) mediante el gasto total de alimento (S/./animal/periodo) dividido por la ganancia total de peso vivo $(\mathrm{kg})$; y el rendimiento de carcasa (RC) mediante el peso de carcasa ( $\mathrm{kg} \times \mathrm{100})$ dividido por el peso vivo al sacrificio $(\mathrm{kg})$.

Para el análisis estadístico se empleó un diseño completamente randomizado con cuatro tratamientos (raciones) conformados por 10 unidades experimentales (animales). Así mismo, se utilizó la prueba de Bartlett para pesos iniciales para determinar la homogeneidad de las unidades experimentales al iniciar el experimento.

\section{Resultados}

El análisis de los pesos vivos iniciales determinó que los cuyes prevenían de muestras homogéneas. El peso final fue ligeramente mayor para $\mathrm{T} 0$, aunque sin diferencia significativa entre tratamientos. Tampoco se encontró diferencia significativa en los pesos de las semanas intermedias entre tratamientos (Cuadro 2). La ganancia de peso varió entre 10.3 y $12.0 \mathrm{~g} /$ día, encontrándose una ganancia de peso mayor para $\mathrm{T} 0$, aunque sin diferencia significativa entre tratamientos dentro de cada semana del estudio.

El mayor consumo de concentrado por cuy fue en T0 (47.2 g), seguido por T3 (45.1 g), T1 (41.2 g) y T2 (38.8 g), pero sin diferencia significativa entre tratamientos (Cuadro 3)

La conversión alimenticia (base seca) considerando solamente el concentrado fue de $3.54,3.40,3.23$ y 3.95 para T0, T1, T2 y $\mathrm{T} 3$, respectivamente, en tanto que la conversión alimenticia para el concentrado más el forraje fue de 6.12, 6.21, 6.03 y 6.91 para T0, $\mathrm{T} 1, \mathrm{~T} 2$ y T3, respectivamente, sin que hubie- 
Cuadro 1. Composición de las raciones experimentales (\%), según la inclusión de frejol Castilla Vigna unguiculata L. Walp)

\begin{tabular}{lcccc}
\hline Ingredientes & $\mathrm{T} 0$ & $\mathrm{~T} 1$ & $\mathrm{~T} 2$ & $\mathrm{~T} 3$ \\
$(0 \%)$ & $(10 \%)$ & $(20 \%)$ & $(30 \%)$ \\
\hline Frejol Castilla & 0 & 10 & 20 & 30 \\
Maíz molido & 56.5 & 48.3 & 46 & 38 \\
Polvillo & 8.1 & 8.5 & 6 & 8 \\
Subproducto de trigo & 7 & 5.6 & 5 & 2.2 \\
Torta de soya & 14 & 10 & 8.5 & 6.2 \\
Pasta de algodón & 11.8 & 14.1 & 12 & 13 \\
Melaza & 1 & 1.6 & 1.1 & 1 \\
Carbonato de calcio & 0.8 & 0.7 & 0.7 & 0.8 \\
Sal común & 0.5 & 0.5 & 0.5 & 0.5 \\
Sales minerales & 0.3 & 0.3 & 0.3 & 0.3 \\
\hline Total & 100 & 100 & 100 & 100 \\
\hline Valor nutritivo & & & & \\
\hline Proteína cruda $(\%)$ & 18.07 & 18.12 & 18.06 & 18.14 \\
E. metabolizable $(\mathrm{kcal} / \mathrm{kg})$ & 3147 & 3127 & 3175 & 3163 \\
Fibra (\%) & 5.1 & 5.21 & 6.53 & 6.84 \\
Calcio (\%) & 0.91 & 0.88 & 0.94 & 1.1 \\
Fósforo (\%) & 0.49 & 0.56 & 0.49 & 0.54 \\
Costo/kg & 1.03 & 0.94 & 0.89 & 0.82 \\
\hline
\end{tabular}

${ }^{1}$ Análisis realizado en el laboratorio de nutrición animal de la Facultad de Zootecnia de la Universidad Pedro Ruiz Gallo

Cuadro 2. Cambios de peso vivo $(\mathrm{g})$ semanal en cuyes mejorados $(\mathrm{n}=10)$ alimentados con cuatro niveles de frejol Castilla (Vigna unguiculata L. Walp) en el concentrado durante la fase de crecimiento-engorde

\begin{tabular}{lcccc}
\hline Semana & $\begin{array}{c}\text { T0 } \\
(0 \%)\end{array}$ & $\begin{array}{c}\text { T1 } \\
(10 \%)\end{array}$ & $\begin{array}{c}\text { T2 } \\
(20 \%)\end{array}$ & $\begin{array}{c}\text { T3 } \\
(30 \%)\end{array}$ \\
\hline 0 (inicial) & $387.3 \pm 26.5$ & $387.8 \pm 41.8$ & $387.6 \pm 44.1$ & $387.4 \pm 47.6$ \\
1 & $433.3 \pm 28.0$ & $440 \pm 34.0$ & $425.9 \pm 34.2$ & $431.2 \pm 42.0$ \\
2 & $511.1 \pm 39.5$ & $492 \pm 30.4$ & $492.3 \pm 46.5$ & $499 \pm 54.3$ \\
3 & $604.7 \pm 52.9$ & $582.6 \pm 31.1$ & $572.4 \pm 47.3$ & $578.6 \pm 62.4$ \\
4 & $703.0 \pm 62.4$ & $679.6 \pm 45.7$ & $674.0 \pm 65.8$ & $663.7 \pm 65.4$ \\
5 & $797.1 \pm 66.9$ & $765.7 \pm 56.3$ & $761.1 \pm 70.2$ & $733.7 \pm 65.3$ \\
6 & $895.6 \pm 81.7$ & $859.2 \pm 66.9$ & $853.4 \pm 80.8$ & $831.3 \pm 69.8$ \\
7 (final) & $973.9 \pm 97.7$ & $922.6 \pm 75.3$ & $914.5 \pm 84.0$ & $891.4 \pm 85.3$ \\
\hline Diferencia respecto a T0 $(\%)$ & -5.27 & -6.07 & -8.47 \\
\hline
\end{tabular}


Cuadro 3. Consumo de concentrado $(\mathrm{g})$ en cuyes mejorados $(\mathrm{n}=10)$ alimentados con cuatro niveles de frejol Castilla (Vigna unguiculata L. Walp) en el concentrado durante la fase de crecimiento - engorde

\begin{tabular}{ccccc}
\hline Semana & $\begin{array}{c}\text { T0 } \\
(0 \%)\end{array}$ & $\begin{array}{c}\text { T1 } \\
(10 \%)\end{array}$ & $\begin{array}{c}\text { T2 } \\
(20 \%)\end{array}$ & $\begin{array}{c}\text { T3 } \\
(30 \%)\end{array}$ \\
\hline 1 & $20.1 \pm 7.9$ & $17.7 \pm 6.6$ & $19.7 \pm 6.5$ & $17.6 \pm 6.6$ \\
2 & $26.6 \pm 6.5$ & $23.5 \pm 6.3$ & $24.8 \pm 6.7$ & $26.9 \pm 6.3$ \\
3 & $43.3 \pm 6.0$ & $39.5 \pm 6.6$ & $34.2 \pm 6.2$ & $40.4 \pm 5.5$ \\
4 & $53.6 \pm 6.2$ & $47.6 \pm 5.9$ & $43.8 \pm 6.3$ & $52.4 \pm 6.1$ \\
5 & $54.6 \pm 7.0$ & $49.0 \pm 6.0$ & $43.9 \pm 5.3$ & $57.3 \pm 6.8$ \\
6 & $65.3 \pm 6.2$ & $54.3 \pm 6.3$ & $51.6 \pm 5.9$ & $58.4 \pm 6.7$ \\
7 & $66.9 \pm 7.4$ & $56.4 \pm 6.6$ & $53.9 \pm 6.0$ & $62.3 \pm 7.0$ \\
\hline Total & 330.3 & 288.0 & 271.9 & 315.4 \\
\hline
\end{tabular}

Cuadro 4. Rendimiento de carcasa en cuyes mejorados $(n=10)$ alimentados con cuatro niveles de frejol Castilla (Vigna unguiculata L. Walp) en el concentrado durante la fase de crecimiento - engorde

\begin{tabular}{lcccc}
\hline & T0 & T1 & T2 & T3 \\
& $(0 \%)$ & $(10 \%)$ & $(20 \%)$ & $(30 \%)$ \\
\hline Peso vivo final $(\mathrm{g})$ & $973.9 \pm 97.7$ & $922.6 \pm 75.3$ & $914.5 \pm 84.0$ & $891.4 \pm 85.2$ \\
Peso de carcasa (g) & $648.9 \pm 65.1$ & $602.5 \pm 49.1$ & $604.4 \pm 55.5$ & $579.1 \pm 55.4$ \\
Rendimiento de carcasa (\%) & $66.6 \pm 17.5$ & $65.3 \pm 20.3$ & $66.1 \pm 18.7$ & $65.0 \pm 18.9$ \\
\hline Diferencia respecto a T0 (\%) & & -7.2 & -6.9 & -10.8 \\
\hline
\end{tabular}

ra diferencia estadística entre tratamientos en ambos casos.

En el Cuadro 4 se presenta el rendimiento de carcasa. Los cuyes del grupo T0 presentaron un ligero mayor rendimiento (66.6\%), pero sin diferencia significativa entre tratamientos. El mayor mérito económico correspondió al grupo T2 $(20 \%$ de frejol Castilla), resultando una mejora del $20.7 \%$ respecto a T0 (Cuadro 5), aunque sin diferencia significativa.

\section{Discusión}

El uso del frejol Castilla en las raciones no afectó el incremento de peso de los animales experimentales. Sin embargo, las ganancias de peso diario y total por cuy fueron superiores a los reportados por Flores (2006), quien evaluó el crecimiento y engorde de cuyes mejorados por nueve semanas comparando raciones alimenticias en altura. No obstante, mayores ganancias de peso fueron reportadas por León (2005), con incremen- 
Cuadro 5. Mérito económico de cuyes mejorados alimentados con frejol Castilla (Vigna unguiculata $L$. Walp) en la fase de crecimiento y engorde

\begin{tabular}{lcccc}
\hline & $\mathrm{T} 0$ & $\mathrm{~T} 1$ & $\mathrm{~T} 2$ & $\mathrm{~T} 3$ \\
& $(0 \%)$ & $(10 \%)$ & $(20 \%)$ & $(30 \%)$ \\
\hline $\begin{array}{l}\text { Ganancia de peso (kg) } \\
\text { Consumo de alimento }\end{array}$ & 0.587 & 0.535 & 0.527 & 0.504 \\
$\quad \begin{array}{l}\text { Concentrado (kg/cuy) } \\
\quad \text { Forraje (kg/cuy) }\end{array}$ & 2.31 & 2.02 & 1.90 & 2.21 \\
$\begin{array}{l}\text { Costo/kg } \\
\quad \text { Concentrado }\end{array}$ & 5.40 & 5.37 & 5.30 & 5.32 \\
$\quad$ Forraje & 1.03 & 0.94 & 0.89 & 0.82 \\
Gasto S/. por cuy & 0.20 & 0.20 & 0.20 & 0.20 \\
$\quad$ Concentrado & & & & \\
$\quad$ Forraje & 2.38 & 1.90 & 1.69 & 1.81 \\
Mérito económico & 1.08 & 1.07 & 1.06 & 1.06 \\
$\quad \begin{array}{l}\text { Total S/. } \\
\quad \text { Solo concentrado }\end{array}$ & & & & \\
\hline Eficiencia respecto a T0 (\%) & 5.89 & 5.55 & 5.22 & 5.7 \\
\hline
\end{tabular}

tos diarios de 11.9 a $13.5 \mathrm{~g}$ en cuyes mejorados alimentados con alfalfa y concentrado.

Menores pesos finales a la séptima semana experimental fueron obtenidos por $\mathrm{Pa}$ lomino (1997) con cuyes mejorados de la provincia de Jaen (659 g), por Cruz (1997) utilizando el aditivo Betafin (640-647 g) y por Cancino (1997) trabajando con diversos niveles de lisina y metionina (667-800 g). Por otro lado, Flores (2006) reporta pesos finales a la novena semana entre 573 y 629 g con dietas en altura.

El consumo de concentrado en cuyes mejorados fue más alto que en otros estudios. Así, se reportan 22.6-26.2, 25.3 y 30 g/cuy/día en los trabajos de Cancino (1997), Palomino (1997) y Cruz (1997), respectivamente.

Los mejores índices de conversión alimenticia se obtuvieron en los tratamientos T2 y $\mathrm{T} 1$ con menores porcentajes de frejol Castilla (20 y 10\%, respectivamente), superando al
T3, que dispuso de $30 \%$ de frejol Castilla en la dieta. La explicación de dicho comportamiento radicaría en la presencia de factores antinutricionales en el frejol Castilla como inhibidores de proteasas, los cuales pudieron incidir de forma negativa sobre la digestibilidad de los nutrientes en los cuyes, disminuyendo así la digestibilidad de las proteínas (Gómez-Villalva, 2005), la digestión y la absorción de nutrientes (Gómez et al., 1998; Martínez y Larralde, 1984) produciendo la hipertrofia del páncreas y una reducción en la energía disponible del alimento (Maynard, 1995) con el consiguiente desmedro en el crecimiento y desarrollo de los animales (Liener y Kakade, 1980).

Similares índices de conversión a los del presente estudio fueron reportados por Saravia et al. (1985), quienes mencionan 3.81 en cuyes alimentados con una ración equilibrada con vitamina $\mathrm{C}$ más agua. Asimismo, León (2005) reporta conversiones alimenticias de 5.95, 5.16, 5.43 en tres tipos de cuyes mejorados, en tanto que Cruz (1997), evaluando 
la inclusión de betaína en la dieta de machos mejorados, reporta conversiones desde 5.05 a 5.52. Conversiones mejores (2.90) han sido reportadas por Tamaki (1972).

En cuanto al rendimiento de carcasa, León (2005) reporta rendimientos de carcasa entre 70.9 y $73.2 \%$ en cuyes mejorados procedentes de Cutervo; de la misma manera, Cerna y Chauca (1997) reportaron rendimientos de 70.9 a $72.7 \%$ de cuyes machos de la línea Perú, alimentados con residuos de cervecería desecada. No obstante, es importante señalar que estos ensayos tuvieron una duración mayor a la de este experimento.

Maynard et al. (1981) menciona que el mérito económico es una forma de representar la adecuación nutritiva de una ración, y que no es permanente en el tiempo, ya que variará en función de los cambios en el precio de los insumos. En el presente estudio, el precio del bajo precio del producto evaluado ( 0.40 soles por kilo), permitió emplear las cantidades expuestas, aminorando de esa manera los costos de alimentación.

\section{Conclusiones}

- El uso del frejol Castilla en reemplazo de otros nutrientes en las raciones de cuyes mejorados no afectó el peso vivo final, la ganancia de peso, el consumo de alimento ni el rendimiento de carcasa.

- El mejor índice de conversión alimenticia se obtuvo al emplear $20 \%$ de frejol Castilla, aunque sin diferencia significativa con los demás tratamientos.

- El mejor mérito económico se encontró al utilizar 20\% de frejol Castilla en la ración.

\section{Literatura Citada}

1. Cancino DI. 1997. Lisina y metionina en la dieta para cuyes, según fase de crianza. Tesis de Ing. Zootecnista.
Lambayeque, Perú: Univ. Nacional Pedro Ruiz Gallo. 75 p.

2. Cerna A, Chauca L. 1997. Evaluación de cuatro niveles de residuos de cervecería seco en el crecimiento y engorde de cuyes. En: Memorias XX Reunión Científica APPA, Tingo María, Perú.

3. Chauca L. 1997. Producción de cuyes (Cavia porcellus). Roma. Italia: Producción y Sanidad Animal, FAO. [Internet]. Disponible en: http:// www.fao.org/docrep/W6562s/ w6562s00.HTM

4. Cruz R. 1997. Acción promotora del betafín en la dieta para cuyes destetados. Tesis de Ing. Zootecnista. Lambayeque, Perú:Univ. Nacional Pedro Ruiz Gallo. 85 p.

5. Cruz A, Ortiz L. 2010. Evaluación de cebada hidropónica (Hordeum vulgare), maíz hidropónico (Zea mays), alfalfa (Medicago sativa) y mezcla forrajera en la alimentación de cuyes (Cavia porcellus), en Antonio Ante, Provincia Imbabura. Tesis de Ing. Ciencias Agropecuarias y Ambientales. Ibarra, Ecuador: Univ. Técnica del Norte. $100 \mathrm{p}$.

6. Fajardo P. 2011. Utilización del factor de transferencia plus tri - fórmula en tres dosis en cuyes hembras gestantes en la granja Producuy - Salcedo - Cotopaxi. Tesis de Médico Veterinario Zootecnista. Latacunga, Ecuador: Univ. Técnica de Cotopaxi. 110 p.

7. Figueroa V, Sánchez M. 1997. Tratamiento y utilización de residuos de origen animal, pesquero y alimenticio en la alimentación animal. Estudio FAO Producción y Sanidad Animal. Roma. Italia. $265 \mathrm{p}$.

8. Flores Q. 2006. Crecimiento - engorde de cuyes mejorados según el nivel de energía y proteinas en su dieta para altura. Tesis de Ing. Zootecnista. Lambayeque, Perú: Univ. Nacional Pedro Ruiz Gallo. 39 p.

9. Gómez-Villalva E. 2005. Trasformación y mejora del valor nutritivo de la harina de guisante mediante la adición de la enzima fitasa. Tesis Doctoral. Granada, España: Univ. de Granada. 222 p. 
10. Gómez G, Vargas R, Quesada S. 1998. Crecimiento y conversión alimenticia de ratas Sprague Dawley sometidas a la ingesta de extractos acuosos de pejibaye (Bactris gasipaes). Agron Costarricense 22: 185-189.

11. Guerra C. 2009. Manual de crianza de cuyes (Cavia porcellus L). Lima, Perú: CEDEPAS Norte. 25 p. [Internet]. Disponible en: http://www.cedepas.org.pe/ sites/default/files/manual tecnico_de crianza_de_cuyes.pdf

12. León E. 2005. Rendimiento de cuyes mejorados de diferente procedencia en Cutervo. Tesis de Ing. Zootecnista. Lambayeque, Perú: Univ. Nacional Pedro Ruiz Gallo. 38 p.

13. Liener I, Kakade M. 1980. Protease inhibitors. In: Liener I (ed). Toxic constituents of plant foodsutffs. USA: Academic Press. p 7-71.

14. Maynard L. 1995. Nutrición animal. $4^{\mathrm{a}}$ ed. México: McGraw-Hill. p 164-167.

15. Maynard L, Loosli JK, Hintz HF, Warner RG. 1981. Nutrición animal. $4^{\mathrm{a}}$ ed. McGraw-Hill. México. 640 p.

16. Martínez J, Larralde J. 1984. Developmental changes on protein turnover in growing rats fed on diets containing field beans (Vicia faba L.) as a source of protein. Arch Latinoam Nutr 34: 465-476.
17. Moncayo R. 2012. Producción de cuyes. Proceso productivo-alimentación. Criadero Auquicuy. Ibarra, Ecuador. $18 \mathrm{p}$.

18. Nacional Research Council (NRC). 1995. Nutrient requirements of laboratory animals. $4^{\text {th }}$ rev ed. Washinton, USA: NRC. 192 p.

19. Palomino FA. 1997. Crecimiento - engorde de tres líneas de cuyes en Jaén. Tesis de Ing. Zootecnista. Lambayeque, Perú: Univ. Nacional Pedro Ruiz Gallo. 62 p.

20. Ramírez M. 2015. Efecto de la utilización de forraje verde hidropónico de Hordeum vulgare consociado a la $\mathrm{Vi}$ cia sativa sobre la ganancia de peso vivo en Cavia porcellus destetados. Tesis de Ing. Zootecnista. Huancavelica, Perú: Univ. Nacional de Huancavelica. 123 p.

21. Rico E, Rivas C. 2003. Manual sobre el manejo del cuy. Provo, Utah, EEUU: Benson Agriculture and Food Institute. $51 \mathrm{p}$.

22. Saravia J, Muscari J, Chauca L. 1985. Prueba de tres niveles de vitamina $\mathrm{C}$ en raciones para cuyes. En: VIII Reunión de la Asociación Peruana de Producción Animal - APPA. Huancayo.

23. Tamaki HR. 1972. Prueba de dos niveles de vitamina $\mathrm{C}$ como posible sustituto del forraje verde en la alimentación de cobayos. Tesis de Ing. Zootecnista. Lima, Perú: Univ. Nacional Agraria La Molina. 45 p. 